\title{
Discours rapporté et mimétique de l'émotion vécue : le cas des soignants en psychiatrie
}

\author{
Louis Maritaud ${ }^{1, *}$ \\ ${ }^{1}$ ED 484 3LA, Université Lumière Lyon 2, Laboratoire ICAR (UMR 5191) 69007 Lyon, France
}

\begin{abstract}
Résumé. L'évolution du système de soin, tournée vers de nouveaux modes de coopération et l'augmentation des intervenants auprès des patients, implique la nécessité pour les soignants de mutualiser l'information concernant le dossier du patient, notamment lors de réunions de relèves. Ce sont 19 enregistrements de réunions de relèves infirmières qui constituent notre corpus d'étude, que nous avons filmées durant 5 semaines dans un hôpital psychiatrique de la région lyonnaise

Lors de ces réunions, une grande partie du discours des soignants est du discours rapporté (DR), au sein duquel les soignants peuvent actualiser un discours premièrement énoncé par quelqu'un, et le rendre ainsi audible à l'ensemble de l'équipe. Selon la forme qu'il revêt, le discours rapporté peut tendre vers une mimétique des propos tenus à l'origine, notamment dans le cas des discours rapportés directs. Dès lors, une certaine forme de mimesis peut être repérée dans les émotions véhiculées par le biais du DR. Qu'il s'agisse de l'expression d'émotions relatives à la pathologie du malade (anxiété, angoisse...) ou d'émotions n'ayant pas de rapport direct avec les troubles pris en charge dans des structures psychiatriques, nous verrons que le $\mathrm{DR}$, dans les réunions de relève infirmière, joue un rôle prépondérant dans l'expression émotive du patient face à l'équipe soignante.
\end{abstract}

\begin{abstract}
The French healthcare system is evolving, impacting the number of caregivers surrounding the patients. Therefore, it happens to be necessary to mutualise information about the patient's files, especially during handovers. 19 recordings of these meetings constitute our corpus, collected during 5 weeks in a psychiatric hospital in Lyon's area.

During these handovers, a large part of the content is delivered in the form of reported speech, thanks to which caregivers are able to make peers hear what they themselves had heard by patients or other caregivers during their shift.. Depending on its form, reported speech can tend to a mimesis of what was said originally, for instance in direct forms. Hence we can spot a kind of mimesis of the emotions which are made explicit thanks to reported speech. Wether it is about emotions specifically related to the patients' sickness or more ordinary emotions, we will see that reported speech, during
\end{abstract}

* louis.maritaud@ens-lyon.fr 
nurses' handovers takes a special place in the expression of the patient's emotions towards the other caregivers.

\section{Introduction}

Une des particularités de l'exercice infirmier en psychiatrie est que ce soignant traite à la fois le somatique et ce qui appartient au domaine de la psychologie. Or, si l'aspect somatique est visible par le biais d'auscultations physiques, l'aspect psychologique demeure la plupart du temps invisible au regard du corps du patient. Bien que des marqueurs cliniques soient repérables dans le comportement des personnes hospitalisées et qu'une médication puisse se révéler nécessaire, une grande partie de l'anamnèse et du soin psychologique se fera donc par le biais du langage.

Afin de maintenir une activation constante de la connaissance des dossiers des patients, les soignants, notamment en psychiatrie, se livrent à des réunions de relève. Dès lors, ces réunions, ayant lieu lors des prises de postes des uns et du départ des autres, sont des moments clés dans le parcours de soins. Nous avons enregistré ces réunions dans un hôpital psychiatrique de la région lyonnaise, durant 5 lundis consécutifs. Dans cette structure, les relèves se déroulent comme suit :

- Indépendamment de l'horaire de la réunion, le schéma protocolaire est toujours le même. Les soignants passent en revue l'ensemble des patients de la structure, en énonçant leur actualité clinique. Pour ce faire, un des soignants est désigné ou s'auto désigne au préalable en tant que responsable de ladite réunion (posture énonciative que nous appelons le « super locuteur »), puis fait le tour des patients présents dans la structure, par numéro de chambre, à l'aide d'une feuille de relève. Sur cette feuille sont notées diverses informations, et il est important de spécifier que si celle du locuteur désigné pour mener la réunion est remplie au préalable par ses soins, ce n'est pas le cas de celles des autres participants qui eux devront la remplir au fur et à mesure de l'interaction, en prenant en note les informations données par l'infirmier passant en revue les actualités cliniques des patients du service. Les autres participants pourront être également sélectionnés ou s'autosélectionner pour prendre la parole, mais la gestion globale de la relève incombe à ce super locuteur.

- $6 \mathrm{~h} 30-6 \mathrm{~h} 45$ et $21 \mathrm{~h} 45-22 \mathrm{~h}:$ les soignants de nuit partent, et transmettent les informations sur les patients aux équipes du matin (et inversement le soir). Lors de ces réunions, seule une partie des soignants est présente, les participants ayant tous comme fonction infirmier ou aide-soignant. Deux soignants sont mobilisés au sein du service afin de procéder à un « tour » des chambres, et s'occuper des patients éveillés

- $9 \mathrm{~h}-10 \mathrm{~h} 30$ : cette réunion, particulière, est nommée " staff hebdomadaire » par les soignants. Réalisée une fois par semaine, elle regroupe une bien plus grande pluralité d'acteurs du service. En effet, y sont conviés les infirmiers et aides-soignants mais également les psychiatres, psychologues, ergothérapeutes, cadres de soins, etc. Cruciale pour le bon déroulement du parcours de soins des patients, il s'agit d'un moment riche d'échanges, dépassant parfois le cadre strict de l'actualité clinique du patient pour s'ouvrir sur des potentielles orientations thérapeutiques par exemple ( $c f$. partie 2.1). Nos analyses seront portées sur une réunion de 9 heures.

$14 \mathrm{~h}-14 \mathrm{~h} 30$ : à l'image de la relève du matin, on ne retrouve ici plus que des infirmiers et aides-soignants, en plus grand nombre du fait du nombre de soignants présents dans l'unité le matin et l'après midi versus la nuit. Les soignants du matin transmettent les informations aux soignants de l'après midi.

Lors de ces réunions, une grande partie du discours des soignants est du discours rapporté (désormais DR), au sein duquel les acteurs du système de soins peuvent actualiser un discours 
attribué lors de sa première énonciation à un tiers énonciatif (c'est-à-dire une personne autre que le « je », hic et nunc. Dans notre corpus, il peut s'agir d'un soignant, d'un patient ou d'un proche du patient par exemple, ayant formulé de propos antérieurement à la relève, qui sont ensuite rapportés lors de celle-ci), et le rendre ainsi audible par l'ensemble de l'équipe. Selon la forme qu'il revêt, le discours rapporté peut tendre vers une pseudo mimétique des propos tenus à l'origine, notamment dans le cas des discours rapportés directs [1]. Dès lors, une certaine forme de mimesis peut être repérée dans les émotions véhiculées par le biais du DR.

Rapporter des propos en discours direct implique l'utilisation du pronom «je » et le respect des temps et déictiques d'origine (par exemple, «Jean a dit "je suis ici" »), ce qui offre un effet de mimétique plus fort que l'utilisation du discours rapporté indirect (« Jean a dit qu'il était là-bas »). Il est important de noter qu'il s'agit seulement d'un effet, et que nous ne nous attachons qu'à étudier cet effet. Les propos tenus en première instance ne sont pas accessibles dans notre corpus (car souvent produits hors des relèves), et donc la fidélité de cette pseudo mimétique ne pourra pas être considérée.

De plus, puisque le discours rapporté implique une pluralité de locuteurs [2], alors le soignant produisant une séquence rapportée subira un décrochement énonciatif afin de placer au cœur de sa propre parole celle du patient ou des autres soignants, tant dans son aspect de locuteur (qui a tenu des propos véhiculant des concepts) que de sujet parlant (qui vit et ressent des émotions). On pourra donc repérer dans ces séquences des éléments relevant de ce qui a été dit et de ce qui a été transmis émotionnellement parlant. On retrouve en effet dans les séquences de DR des séquences porteuses d'émotions, qui dépassent le cadre strict des propos tenus pour aller vers la mutualisation des ressentis des patients et des soignants évoqués dans des contextes hors relèves.

Ainsi, nous proposons de décrire le rapport entre la pseudo mimétique du DR et les émotions que les soignants mutualisent lors de leurs réunions de relève, des points de vue verbal et gestuel en utilisant les outils de l'analyse conversationnelle. Dans notre corpus, une pluralité de marqueurs prosodiques et gestuels sont repérables dans les séquences de DR, à l'image de ce qui est montré dans les travaux de Morel concernant la prosodie [3], ou de Caillat concernant la gestuelle [4]. Nous pourrons constater que l'expression des émotions joue un rôle fort dans l'expression émotive notamment du patient face à l'équipe soignante.

\section{Cadre théorique}

Nous nous situons dans une perspective conversationnaliste, au sens de Sacks, Schegloff et Jefferson [5], de Traverso [6], Petitjean \& Pekarek Doehler [7] ou encore de Mondada [8, 9], et étudions un phénomène syntaxique et énonciatif, le discours rapporté à l'oral. Nous nous appuierons à la fois sur des travaux en analyse du discours $[4,10]$ que sur des travaux menés en analyse conversationnelle [11,12] pour construire notre cadre théorique sur le DR. Voyons d'abord quelles sont les caractéristiques du discours rapporté (1.1), puis nous discuterons de l'analyse conversationnelle et des perspectives qu'elle offre dans le traitement des aspects paraverbaux, c'est-à-dire de la multimodalité (1.2).

\subsection{Le discours rapporté}

Le DR est une forme d'actualisation d'un discours issu d'une autre situation d'énonciation, formulé ou non en première instance, provenant d'un individu réel ou fictif. Étudié depuis longtemps dans des corpus écrits, l'intérêt des perspectives sur les séquences de DR oral est plus récent. Dans les deux systèmes (oral et écrit), certaines typologies, contraintes et considérations sont cependant communes. Nous verrons tout d'abord quelques distinctions formelles, puis nous parlerons de l'aspect pseudo mimétique des séquences de DR. 


\subsubsection{Distinctions formelles}

Traditionnellement, le DR est considéré selon le degré d'inclusion du discours cité dans le discours citant [10]. En effet, on peut distinguer le discours rapporté direct (désormais DRD) du discours rapporté indirect (désormais DRI). Ces deux catégories de DR ont d'abord été décrites à l'écrit selon des critères grapho-syntaxiques, et possèdent des sous-catégorisations que nous n'explorerons pas dans cet article, du fait de la difficulté à les appliquer à l'oral. Le DRD se caractérise par une non-variation des déictiques et du temps utilisé dans le discours cité, alors que le DRI subira une modification de ces derniers. Au DRD, on pourra donc dire "il a dit je suis ici maintenant", tandis qu'au DRI, on dira "il a dit qu'il était là-bas à ce moment là" par exemple.

Ces distinctions entraînent une intégration plus ou moins importante du discours cité dans le discours citant. En effet, un décrochement énonciatif (c'est-à-dire un changement de posture énonciative, résultant du passage du discours du locuteur au discours rapporté) plus ou moins marqué sera repérable dans les séquences citées, en partie selon les paramètres précédemment mentionnés. Ainsi, les séquences de DR peuvent être considérées comme relevant d'un continuum d'intégration des propos tenus en première instance dans ceux formulés hic et nunc, et au sein de cette intégration relative, nous pouvons observer une pseudo mimétique plus ou moins marquée.

\subsubsection{Perspectives mimétiques}

Rapporter un discours tiers à l'oral implique de mettre en scène plus que du verbal. Qu'il s'agisse d'un contenu informationnel, d'un lien énonciatif du locuteur citant avec les propos du locuteur cité ou simplement des caractéristiques associées au locuteur cité, on peut souvent remarquer un réel balisage en faisceaux multimodaux tendant vers une pseudo mimétique, à l'image de l'intégration plus ou moins importante du discours cité dans le discours citant précédemment explicitée. Ainsi, nous pourrons observer des variations prosodiques [3], gestuelles [4] ou stylistiques pouvant tendre vers la représentation du locuteur auquel les propos rapportés dans les séquences de DR sont attribués en première instance.

Il convient de distinguer deux aspects ici. D'une part, la mimétique peut être de l'ordre du verbal, c'est à dire relever de critères syntaxiques, lexicaux et grammaticaux. Dans le cadre du DRD par exemple, l'utilisation du " je » référant au locuteur représenté tend à produire une pseudo mimétique plus forte des propos rapportés. On attribuera alors le message au locuteur tiers dans son ensemble, soit sa forme et son contenu, tandis que dans le cadre du DRI, on attribuera plus la forme mais seulement le contenu du message au locuteur représenté.

Du point de vue multimodal (gestuelle, postures, prosodie), les variations entre discours citant et discours cité pourront également tendre vers une pseudo mimétique, mais pas seulement. En effet, dans un effort de manifestation d'un décrochement énonciatif, d'un lien du locuteur « je " hic et nunc vis-à-vis des propos rapportés, ou même pour exhorter l'audience à avoir une réaction, diverses manifestations seront possibles. Pour n'en citer que quelques-unes, les participants auront tendance à porter le regard ailleurs que sur l'interlocuteur lors des séquences de DR [4], à hausser la fréquence fondamentale de la voix sur les séquences introductives du DR (" il a dit que » par exemple, que nous nommerons désormais inquit), ou encore à avoir une hausse conjointe sur les propos rapportés (que nous nommerons portée du DR) dans la fréquence et l'intensité vocales [3].

Ces séquences ne surviennent pas isolément. Elles font partie d'un ensemble conversationnel, dans un contexte particulier, ici celui de la relève infirmière. Pour pouvoir appréhender cet aspect global, nous nous concentrons sur les outils de l'analyse conversationnelle, que nous allons désormais expliciter. 


\subsection{L'analyse conversationnelle}

Née dans les années 1960 avec les travaux de Sacks, rejoint rapidement par Schegloff et Jefferson, l'analyse conversationnelle est issue du courant de l'ethnométhodologie de Garfinkel. Elle permet d'aborder de façon rigoureuse l'organisation sociale de l'action, et est particulièrement utile dans notre cas pour appréhender le discours en situation professionnelle, ainsi que ses aspects multimodaux.

\subsubsection{Les interactions en situation professionnelle}

Un regard particulier s'est porté sur les interactions en situations professionnelles pour l'analyse conversationnelle. En effet, cette dernière porte « une attention pour l'organisation du langage et de l'action au sein de l'interaction sociale, tels qu'ils s'y organisent de manière localement située » [8, p.181], et dans un cadre professionnel, les interactions sont gérées différemment. Nous pouvons ici citer Mondada \& Keel [13], décrivant l'asymétrie dans des situations professionnelles entre profanes et experts. Des ressources traitant la question de la relève infirmière existent, et nous pouvons par exemple citer Grosjean $[14,15]$ et Grosjean \& Lacoste [16], s'étant notamment attachées à décrire les situations de réunions de transmission d'informations du point de vue conversationnaliste, montrant par exemple leur caractère très organisé. Dans notre cas, la relation entre les participants n'est pas du même type, chacun ayant le même statut expert quant à la pratique des soins infirmiers. Cependant, nous avons pu voir dans des analyses précédentes des distinctions entre phases de " faire apprendre » et « faire savoir », représentées notamment au niveau gestuel [17].

\subsubsection{La multimodalité}

L'intérêt porté pour les aspects non verbaux du langage s'est transformé en analyse scientifique rigoureuse et en point central de perspectives de recherches de façon relativement récente. Dès lors, une approche plus systématique des paramètres paraverbaux s'est mise en place, comme les regards [18, 19], postures [11], gestes [20, 21] par exemple. Aujourd'hui, il s'agit de l'un des points d'ancrages de l'analyse conversationnelle et d'autres courants théoriques, la multimodalité étant par essence abordée d'un point de vue pluridisciplinaire [22]. Nous nous attacherons dans cet article à décrire la gestuelle des participants, et de la contextualiser avec l'émotion transmise (notamment du point de vue lexical) dans les séquences de DR produites. Ainsi, nous pourrons voir se dessiner un continuum suivi par plusieurs aspects : la structure grammaticale des séquences, la gestuelle des participants et l'émotion lexicale contenue dans les séquences de DR analysées.

\section{Analyses}

Nous analyserons dans cette partie deux séquences, l'une dans laquelle le verbal, l'émotion et la gestuelle tendent simultanément vers une pseudo mimétique forte, et l'une dans laquelle ce n'est pas le cas. Les deux extraits se déroulent lors de la même réunion, et il s'agit ici du staff médical, regroupant donc des soignants de fonctions diverses : $\mathrm{GAL}^{\dagger}$ est psychiatre, POM est psychomotricienne, LAR est assistante sociale, ZAC est étudiant en Institut de Formation en Soins Infirmiers, LIM est interne en psychiatrie, et PAC, GIL, PAT sont infirmières. Il est important de préciser que GIL est lors de cette réunion la super locutrice, à qui incombe donc la responsabilité du bon déroulement de la relève (elle passe en revue

\footnotetext{
† Tous les noms sont des pseudonymes créés à partir de noms d'emprunt
} 
l'actualité clinique de tous les patients, gère la séquentialité de la réunion, l'ouvre et la clôture). Dans notre cas, nous étudierons les gestes de PAT, puis de GIL, toutes deux infirmières.

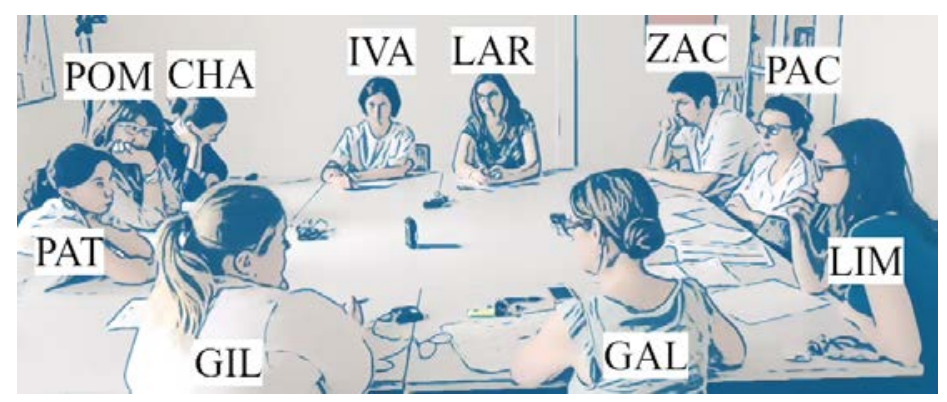

Image 1. Disposition des participants à la réunion analysée

Voyons donc en premier lieu des séquences de discours rapporté dans lesquelles la syntaxe, la gestuelle et l'émotion suivent un continuum commun, c'est-à-dire des cas dans lesquels la pseudo mimétique est similaire du point de vue syntaxique et gestuel, avec une émotion transmise plus ou moins forte selon ce degré de pseudo mimétique.

\subsection{La gestuelle mimétique est congruente à la mimétique syntaxique : « le temps en chambre »}

Ce premier extrait long, que nous analyserons en trois temps, se situe à la fin de la relève concernant le patient dont les soignants parlent. Il y est question de la mise en place d'un séjour en ESPI, Espace de Soins Psychiatriques Intensifs, permettant un isolement du patient, au bénéfice de sa thérapie. Nous pourrons y voir PAT exposer une idée afin de négocier l'isolement du patient. En effet, ce dernier ayant des discours et des réactions divergentes face à ce genre de thérapie, les soignants cherchent ici à tabler sur la marche à suivre dans la continuité du parcours de soin et de l'hospitalisation. Si la véritable décisionnaire est GAL, psychiatre, les autres participants à la relève sont entendus, et leurs arguments sont pris en compte. Ainsi, les soignants peuvent exposer leur vécu et celui du patient pour aider GAL à décider quelle option thérapeutique sera la meilleure pour le patient. Pour ce faire, PAT produira plusieurs séquences de DR, rapportant tantôt les propos du patient, tantôt ceux des soignants, véhiculant un discours plus ou moins chargé d'émotions lexicalement et paraverbalement, utilisant des formes de DR et une gestuelle variées.

\section{Extrait 1 :}

1 PAT après au pire si il supporte pas 1'temps-' $\mathrm{fin}$ si

2 le côté fermé euh est-ce que lui dire au moins

9 GAL $\quad[\mathrm{hm} \quad]$ 
10 GIL hm

11 POM oui peut [être si à mon avis on devrait tenter ]

12 PAT [ $j$ sais pas si- est ce que ça pourrait]

13 p't être euh:\ou pas marcher mais euh $\backslash$

$14 \mathrm{GAL}$ on peut [tenter ]

15 PAT [parce qu'il] disait la s'maine dernière

16 ouais le salon $j$ 'en veux pas $j$ vais tout péter

$17 j$ vais démonter vot salon si j'y vais/

18 POM oui pis on a [l'impression que (inaud.) ]

19 PAT [et on $\mathrm{s}$ 'était dit est ce que:/euh:\]

20 POM intermédiaire

21 PAT parce que $i-i l$ a dit non non j'irai pas/ euh: $\backslash$

$22 j$ dors en- $j$ reste en chambre $j$ dors en chambre

$23 j$ dormirai dans ma chambre/ et au final il s'est

24 endormi dans sa chambre

25 GAL et oui c'est c' qu'il a fait la s'maine dernière

Nous analyserons tout d'abord la séquence de DR présente lignes 2-8 représentant un potentiel discours soignant non formulé en première instance, puis celle lignes 15-17 rapportant un discours patient lexicalement chargé d'émotion, et enfin celle ligne 21-23, toujours attribuable au patient en première formulation, mais non chargé d'émotion. Nous ajouterons également une transcription multimodale aux extraits analysés, celle ci-dessus ayant vocation de faire transparaître la séquentialité de l'extrait.

\subsubsection{Cas d'un DR attribué à l'équipe}

Dans l'extrait à suivre, PAT va proposer à l'équipe soignante une prescription d'isolement thérapeutique atténuée, prenant la forme d'une séquence de DR d'abord indirect puis direct, et sa gestuelle va suivre la même transition vers une mimétique plus forte.

\section{Séquence 1 :}

pat

le@ côté fermé euh est-ce que lui dire @\#au moins@ aregard table

eregard

GAL- $->5$

Qhausse les

sourcils et hoche modérément la tête@

3

@par [défaut ] ] un\&

pat @hoche la tête modérément @

4 GAL

[un temps en chambre]

5 PAT

pat $\& @[$ temps en CHAMBRE ]

6 GIL

aregard GIL, hoche la tête plus fort \#3

7 PAT

[des temps en chambre] ouais/

pat

et de lui dire @c'est un @cadre c'est @prescrit 


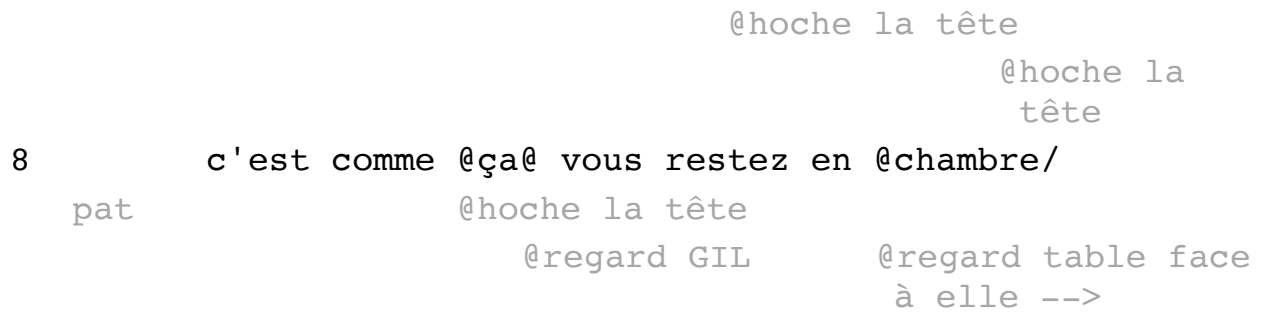

Dans ce premier extrait, PAT produit un long énoncé rapporté (lignes 2 à 8 ), non formulé en première instance, ne présentant pas de contenu lexical émotionnel et représentant une parole soignante hypothétique. Ainsi, si le patient ne supporte pas « le côté fermé » (ligne 2), PAT propose de modifier la prescription pour ce malade et de lui imposer un temps en chambre afin de créer un parcours de soins adapté à sa personnalité et ses problématiques (" c'est un cadre c'est prescrit c'est comme ça » lignes 7-8), l'ESPI précédemment explicité ne convenant pas vraiment à ce patient, pour qui les soins intensifs, et donc l'isolement, sont pourtant nécessaires. Du point de vue gestuel, PAT alterne entre des regards orientés vers GIL, super locutrice en charge de cette relève, et GAL, psychiatre présente durant cette réunion.

Cette alternance du regard montre une hiérarchisation des soignants autour de la table. En effet, le seul pouvoir décisionnaire quant à la prescription pour un patient revient à un médecin, ici GAL, et le statut particulier de super locutrice attribué à GIL en amont de la relève expliquent cette alternance. Par ailleurs, on peut remarquer que ces moments d'alternance portent sur des éléments n'ayant pas la même valeur soignante. Ligne 2, PAT oriente son regard vers GAL lorsqu'elle dit « au moins » (sous-entendu « pas plus que »). On se situe ici dans une négociation active de la prise en charge et la prescription du patient. Lorsque ligne 5 PAT regarde GIL, infirmière, le statut des propos tenus n'est plus le même : il s'agit d'une pratique active de la fonction infirmière, à savoir la vie quotidienne du patient qui, selon la proposition formulée par PAT, se retrouverait en chambre. On s'extrait de la prescription, à la charge du psychiatre (où PAT regarde GAL), pour entrer dans la réalisation active de cette dernière, à la charge des infirmiers (où PAT regarde GIL).

Il est intéressant de noter les hochements de tête successifs de PAT, appuyant les propos ici rapportés. On peut effectivement constater qu'elle hoche la tête sur deux segments présentant la même structure (au moins - par défaut - un temps en chambre, lignes 2 à 5 et c'est un cadre - c'est prescrit - c'est comme ça lignes 7-8), séparés par une incise rappelant que l'on se situe dans une séquence de DR « et de lui dire » ligne 7. Ainsi, on peut observer trois hochements de tête avant cette incise (sur " au moins » ligne 2 (image 2), sur " par défaut » ligne 3 et sur « temps en chambre » ligne 5 (image 3 ), qui est plus accentué que les deux précédents et fonctionne conjointement avec la saillance perceptuelle de « chambre »). Après l'incise, nous pouvons remarquer trois nouveaux hochements de tête sur " cadre ", " prescrit » et « ça » lignes 7-8. Ainsi, on peut remarquer une structure gestuelle se répétant : [inquit] puis 3 hochements de tête, rythmés, que l'on peut expliciter par le fait qu'il s'agit de deux listes tripartites, "phénomène empiriquement observable et récurrent » [23, p.66]. De plus, cette séquence s'ouvre et se ferme sur un regard orienté vers la table, balisant ainsi la séquence de DR en plus des manifestations verbales sous forme d'inquit. 


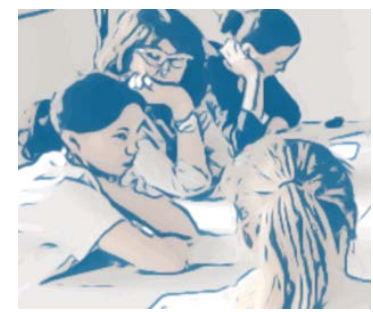

Image 2 : hochement \#2 et regard GAL

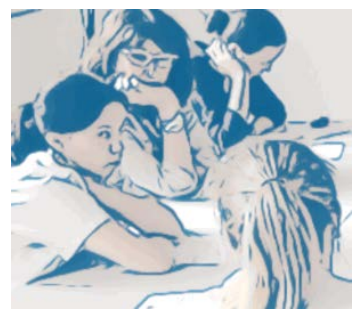

Image 3 : hochement \#3 et regard GIL

Il est également important de noter qu'avant l'incise, la séquence de DR est de type indirect, tandis qu'après, elle devient de type direct. Lors de la transition du DRI au DRD, on peut observer une augmentation de la gestualité de la participante : les hochements de tête se font plus rapprochés et plus marqués. Cette variation tend à nous faire penser que le continuum gestuel suivrait le continuum d'intégration syntaxique du discours cité (donc les propos rapportés) dans le discours citant. Nous pouvons donc voir que la gestuelle occupe une place importante dans les séquences de DR sans contenu émotif exprimé, et peut participer à l'intégration plus ou moins forte de la portée (c'est-à-dire les propos rapportés en tant que tels) dans le reste du discours (lignes 7-8, la gestuelle et plus marquée qu'auparavant, tout comme la pseudo mimétique syntaxique du DRD de ces lignes est plus forte que celle du DRI lignes 2-5). Voyons désormais si, lorsque la portée du DR évoque des émotions, la gestuelle s'en voit impactée.

\subsubsection{Cas d'un DR attribué au patient et chargé d'émotions}

Cette deuxième séquence présente une argumentation active de PAT, appuyant sa proposition précédente (séquence 1) qui revêt la forme d'un DRD exprimant des émotions du patient face à la potentielle prescription du salon d'apaisement, c'est à dire l'Espace de Soins Psychiatriques Intensif, étant ici une chambre permettant l'isolement du patient le plus complet.

\section{Séquence 2 :}

15 PAT pat

16

19 PAT pat

\section{pat}

17

pat

18 POM

parce qu'il @disait la\# s'maine@@dernière\#

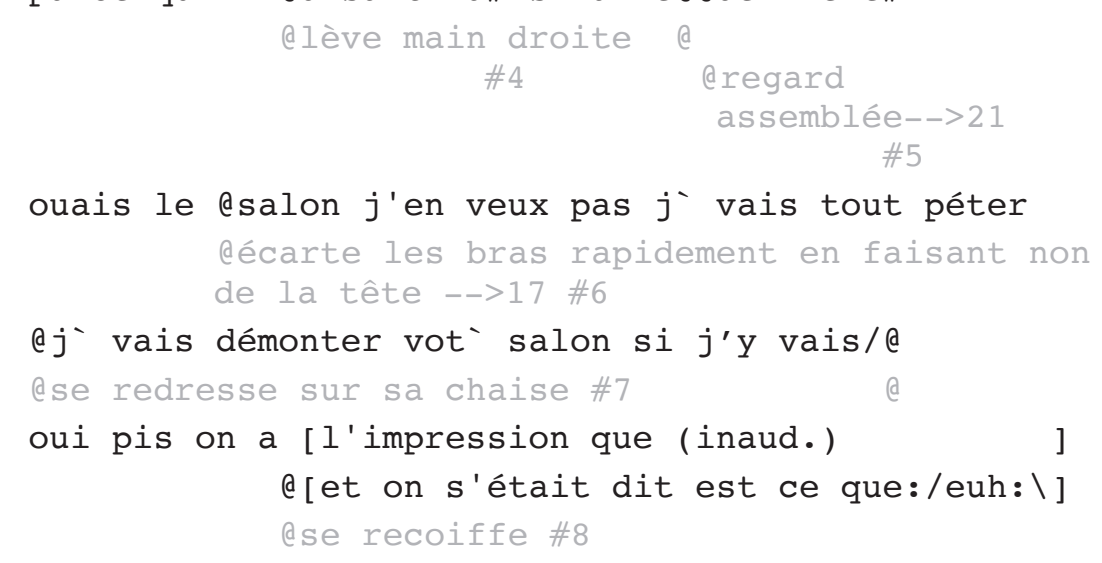
\#4 aregard 
Beaucoup plus courte que la séquence précédente, celle-ci nous présente un DR représentant le patient, dans sa dimension émotive. Ainsi, si les propos du patient sont représentés, les émotions véhiculées par ce biais le sont aussi. On peut en effet citer «j`vais tout péter » ligne 16 , et « $\mathrm{j}$ ' vais démonter vot' salon » ligne 17 , faisant montre d'un état avancé d'agitation du patient face à la proposition faite précédemment de son entrée dans le salon, « la semaine dernière » (ligne 15). Nous avons ici une séquence de DRD, que l'on peut qualifier ainsi au vu de la non-variation des pronoms, « je » (lignes 16-17) référent ici au patient, et pas à PAT qui est le sujet parlant.

Du point de vue gestuel, ligne 15, PAT lève la main droite puis regarde l'assemblée. Elle passe ici d'une posture renfermée, bras croisés sur la table, à une posture plus ouverte, droite (image 4). Son regard, auparavant porté sur GIL ou GAL, parcourt désormais l'assemblée, montrant un statut particulier de cette séquence vis-à-vis de la précédente : là où l'adressage se voulait orienté vers deux participantes, ici l'adressage se veut plus étendu (image 5). Par ailleurs, en s'étant repositionnée, PAT peut avoir une gestuelle potentiellement plus étendue que lors de l'extrait précédent, en incluant des mouvements de bras, à l'image de son mouvement de main ligne 15. Ligne 16, en débutant la portée du DR chargé d'émotion, PAT écarte les bras rapidement en faisant non de la tête, appuyant le refus manifeste verbal du patient par une gestuelle mimétique. Elle présente le désaccord fort du patient avec sa prescription en représentant conjointement un refus verbal et multimodal, dans une séquence de DR (image \#6).

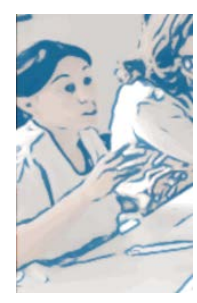

Image 4

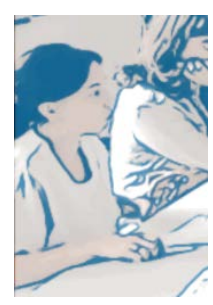

Image 5

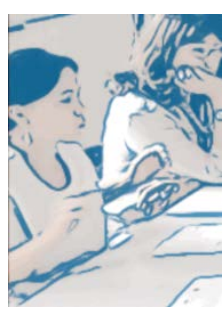

Image 6

Suite à ce DR avec une forte gestualisation, ligne 17, PAT se redresse sur sa chaise, amorçant ainsi la fin de la portée (image 7). En effet, cette action se termine en même temps que l'énonciation des propos attribués au patient en première instance, suite à quoi PAT fait une nouvelle séquence de DR, représentant cette fois l'équipe soignante et ses réactions face à l'événement problématique du refus manifeste et présenté comme fortement marqué du patient d'aller en salon d'isolement (ligne 16). Ainsi, on peut considérer que la séquence gestuelle de représentation des émotions du patient se clôt avec ce changement de posture, et le balisage multimodal de la séquence de DR est complet : l'inquit est marqué par la main droite qui se lève (ligne 15), le refus du patient par la gestuelle mimétique du refus du patient (ligne 16), et la clôture de la séquence par le redressement de la participante (ligne 17), et la représentation de la réponse des soignants démarre conjointement avec la remise en place de ses cheveux par la soignante (ligne 19, image 8). 


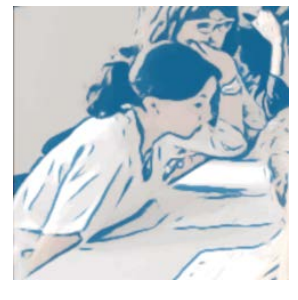

Image 7

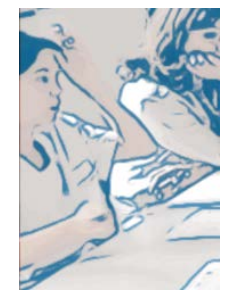

Image 8

On peut donc observer dans cette deuxième séquence chargée d'émotion deux choses. D'une part, PAT gesticule plus que lors de la première séquence dans laquelle l'émotion était absente et d'autre part le balisage multimodal du DR se voit amplifié : PAT réalise une véritable séquence gestuelle à portée mimétique et la clôt en se redressant, pour ensuite changer de locuteur représenté dans une autre séquence de DR. Celle-ci, présentant un discours toujours attribué au patient en première instance, n'est pas chargée d'émotion, mais toujours sous forme de DRD. Voyons ce qu'il advient de la gestuelle de la soignante lorsqu'elle rapporte ces propos.

\subsubsection{Cas d'un DR attribué au patient sans émotion dite}

Cette troisième séquence fait suite à la précédente, et PAT y rapporte d'autres propos attribués au patient, centrés cette fois non plus sur le salon d'isolement, mais sur sa propre chambre, dans laquelle il veut rester. Ici, l'émotion n'est plus exprimée verbalement, mais de façon cotextuelle, à mettre en relation avec la séquence précédente de DR. Une nouvelle fois, nous nous concentrerons sur sa gestuelle et l'intégration syntaxique des propos cités dans le discours citant.

Séquence 3 :

21 PAT parce que $i-i l$ a dit anon none j'irai pas/ euh: $\backslash$
pat
aredresse rapidement la tête,
hause les sourcils, écarquille
les yeuxe \#9
eregard table-->23

22

aj dors en- $\mathrm{aj}^{\mathrm{j}}$ reste en chambre $\mathrm{aj}^{\mathrm{j}}$ dors en

pat aredresse rapidement la tête \#10

aredresse rapidement la tête

@redresse

rapidement la

tête \#11

23

chambre @j dormirai dans ma chambre/

pat

aredresse rapidement la tête

24

let au final il s'est endormi dans sa chambre

pat aregard BER, oriente ses paumes vers le haut puis vers le bas-->

Tout d'abord, nous pouvons observer que le DR est ici direct. En effet, PAT emploie un « je » faisant référence au patient (lignes 21 à 23). Les propos rapportés sont donc soumis à une pseudo mimétique syntaxique forte. Au niveau gestuel, nous pouvons remarquer que le regard de la soignante se porte, tout au long de la séquence rapportée, sur la table. Ce faisant, elle accentue les verbes en redressant rapidement la tête sur « reste » et « dors » ligne 22 
(images 9 et 10). Ces mouvements de tête peuvent être considérés comme mimétiques de la volonté forte du patient de rester dans sa chambre, et être mis en parallèle avec les reformulations/répétitions manifestées dans la séquence (il s'agit ici de l'alternance entre les verbes « dors », " reste » et « dormirai » lignes 22-23, formant une nouvelle liste tripartite [23]).

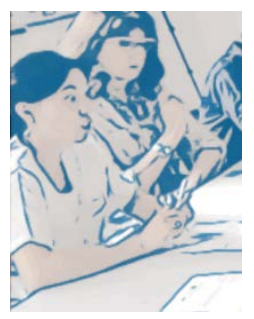

Image 9

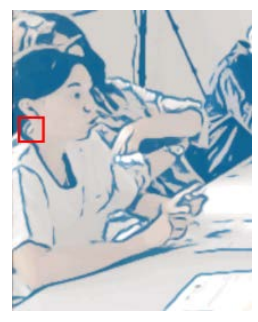

Image 10

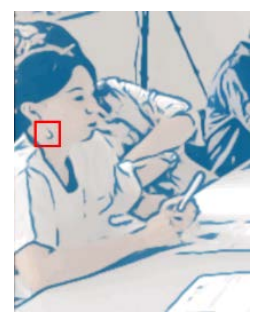

Image 11

Dans les images 10 et 11 les redressements rapides de la tête de PAT sont particulièrement visibles grâce à l'orientation de sa boucle d'oreille, encadrée en rouge. De plus, on peut observer une gestuelle faciale particulièrement accentuée au tout début de la portée de cette séquence, que l'on peut une nouvelle fois mettre en lien avec une mimétique des propos rapportés, et donc de l'état du patient au moment de la première énonciation de la portée du DR en question (image 9). Ici, la soignante ne se contente pas de rendre audible à un auditoire étendu des propos tenus précédemment par le patient. Elle actualise toute une situation d'énonciation, en faisant l'effort de produire une pseudo mimétique de la portée du discours rapporté, mais également de la façon dont ce discours avait été tenu, notamment au niveau des expressions faciales et des gestes.

Nous avons donc pu voir dans l'analyse de ces trois séquences un fonctionnement gestuel congruent à la dimension émotive des propos tenus. La première séquence, rapportant des propos de soignants potentiellement à venir, avait une gestuelle très modérée, la soignante étant les bras croisés sur la table ; la troisième, faisant vivre à un auditoire étendu une situation vécue par une seule soignante, mais sans portée émotive, montrait une gestuelle majoritairement faciale et au niveau de la tête. La deuxième, enfin, présentait une véritable combinatoire entre intégration syntaxique (DRD), gestualisation forte (mains et tête), et balisage multimodal de la séquence rapportée. En symbolisant ces trois séquences sur un continuum, nous nous trouvons avec le schéma suivant, présentant de gauche à droite la première séquence, la troisième puis la deuxième : 


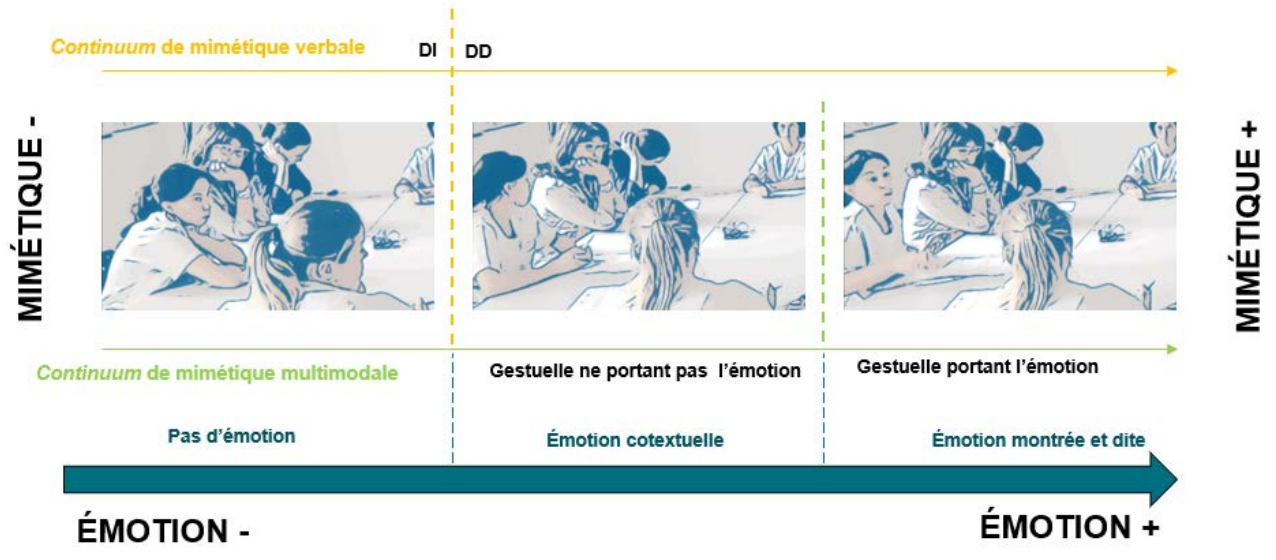

Schéma 1 : Parallélisme entre mimétique gestuelle et verbale, en relation avec la dimension émotive du DR

Nous avons donc pu observer une forme de parallélisme entre la portée émotive des DR, l'intégration syntaxique du discours cité dans le discours citant et une forme de mimétique gestuelle des propos rapportés. Voyons désormais un exemple dans lequel la gestuelle n'est pas congruente à la syntaxe et à la portée émotive des propos tenus.

\subsection{La gestuelle mimétique n'est pas congruente à la mimétique syntaxique : " la fête des mères "}

L'analyse de l'extrait à suivre diffère de ce que nous avons pu voir précédemment. En effet, là où les séquences précédentes montraient clairement un continuum parallèle, dans celle à venir, GIL, rapportant le discours du même patient quelques minutes après l'extrait précédent, produit une autre séquence de DR, mais sans gestualisation forte alors que l'aspect émotif y est très présent au niveau lexical. Nous nous concentrerons sur la gestuelle de GIL, super locutrice de la relève et rapportant les propos du patient. Il convient de préciser que dans cet extrait, GIL prend la parole en tant que soignante, au même titre que les autres participants à la réunion. En effet, si le rôle de super locutrice lui fait porter une charge supplémentaire, elle aussi peut partager son vécu, au même titre que ce que PAT a pu faire dans les extraits précédents. Les soignants étant ici dans une négociation de la prise en charge du patient, GIL intervient donc en tant qu'infirmière, et pas en tant que super locutrice.

\section{Extrait 2:}

\section{((au début de l'extrait, GIL regarde GAL))}

1 GIL Qaprès il faisait quand même beaucoup de menaces un

gil dtourne la tête vers l'assemblée et pose sa main gauche sur son menton-->5

2 peu au @suicide aussi@ alors ce qui l'a quand même
gil

3

4 GAL pas mal [fait ] grimper aussi

5 GIL [ah bon//] 


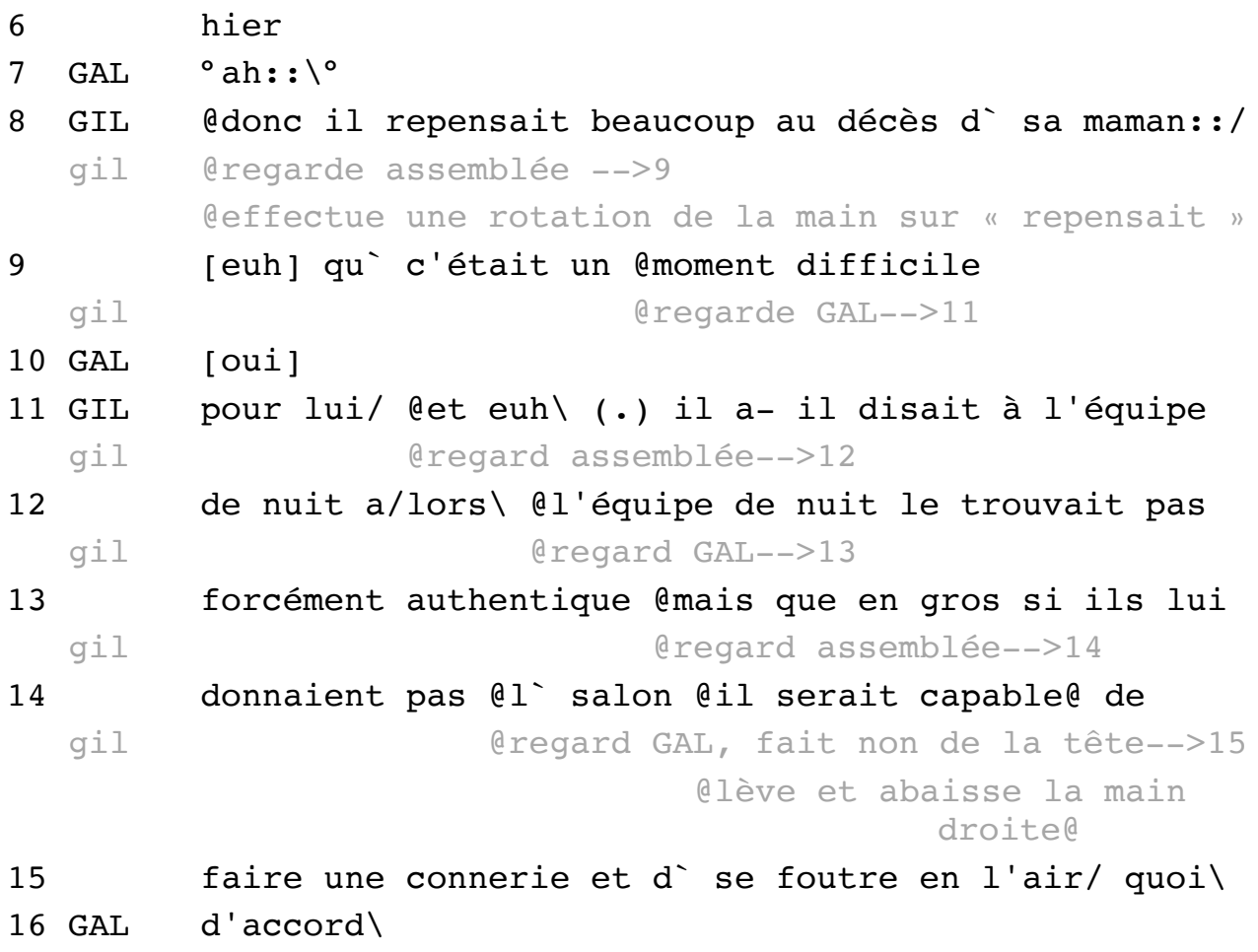

La séquence de DR stricto sensu apparait lignes 11-15, avec une incise lignes 12-13 sur la réception de l'information par « l'équipe de nuit». On peut de prime abord remarquer que la gestuelle de la participante est assez restreinte : elle oriente son regard vers l'assemblée ou vers la psychiatre, réajuste sa position et effectue seulement deux mouvements de main durant cet extrait. Il est important d'entrer dans une dimension comparative ici : si les analyses précédentes montraient ou disaient une forme d'émotion vécue par le patient, ici le cas est critique, la question du suicide étant abordée (ligne 1-2, « menaces au suicide » et ligne 15, « faire une connerie »).

Nous considérons ici cette séquence comme restituant une émotion vécue par le patient pour plusieurs aspects. Si la soignante explicite très clairement une crise suicidaire du patient, on peut aussi remarquer la mention d'un « moment difficile » (ligne 9) auquel le patient « repensait beaucoup » (ligne 8), et qui l'a potentiellement « fait grimper » (ligne 3). Néanmoins, ces moments forts d'informations relevant de l'émotion du patient sont nuancés par des aspects à la fois lexicaux (« des menaces un peu au suicide quand même » ligne 2, «l'équipe de nuit le trouvait pas forcément authentique », ligne 13), et énonciatifs : cette séquence de DR est une représentation d'une séquence rapportée au préalable : les soignants de nuit ont transmis aux soignants de jour leur propre vécu, leurs ressentis face au patient, et les propos tenus par ce dernier, et c'est désormais une infirmière de jour qui transmet ces informations. Ce double DR entraîne de façon assez logique une prise de distance de la part de GIL, tout comme le fait qu'elle n'a pas pu vivre le moment qu'elle rapporte in situ. $\mathrm{Ne}$ l'ayant pas vécu, elle ne peut pas reproduire une gestuelle (ou du moins ne pourrait que reproduire celle des soignants lui ayant rapporté la scène).

Dès lors, deux facteurs s'opposent dans cet extrait, qui n'existaient pas dans les précédents : d'une part l'aspect émotif est très fort et important dans la poursuite des soins, mais d'autre part le moment n'a pas été vécu directement par la soignante qui parle, mais lui a été rapporté. Ainsi, la faible gestualisation du DR peut résulter de cet enchâssement de discours. Par 
ailleurs, on peut remarquer que du point de vue informationnel, GIL négocie activement les propositions préalables de PAT : si un isolement moins fort peut être profitable au patient, et qu'il refuse parfois d'aller en salon d'apaisement, il lui arrive également de menacer les soignants de commettre un suicide si ces derniers n'accèdent pas à sa demande d'aller en isolement.

\section{Conclusion}

Les situations de réunion de relève en soins psychiatriques sont complexes, font intervenir une multitude de participants, avec des buts évoqués (soit transmettre des informations sur l'actualité clinique des patients), et d'autres survenant au cours de la conversation (comme par exemple la prise en charge du patient dans nos extraits). Au sein de ces moments d'échanges, les soignants pourront produire des séquences de DR, notamment pour véhiculer une forme d'émotion vécue par le patient ou les soignants, et la rendre visible par le biais de la gestuelle aux autres participants. Nous avons pu voir que l'intégration syntaxique pouvait correspondre à une pseudo mimétique posturo-gestuelle dans certains cas, notamment dépendant du degré d'émotion contenue dans la portée des séquences de DR, mais que ce constat reste à explorer au vu de certains exemples, que l'on peut expliquer par certains facteurs extérieurs à la relève en elle même ou à l'émotion contenue dans les propos rapportés. De la même façon, une analyse rigoureuse et systématique de notre corpus du point de vue de l'émotion et du DR serait nécessaire pour pouvoir apporter des résultats généralisables et probants. Enfin, il convient également de considérer de façon plus rigoureuse les problématiques n'ayant pas trait à la portée du DR en tant que tel : puisque le balisage multimodal de ces séquences peut se réaliser ou non, alors la corrélation entre gestualisation accentuée et un phénomène particulier reste à démontrer.

\section{Bibliographie}

1. L. Rosier, Le discours rapporté en français. (Ophrys, Paris, 2008)

2. O. Ducrot, Le Dire et le Dit. (Éditions de Minuit, Paris, 1984)

3. M.-A. Morel, Le discours rapporté direct dans l'oral spontané. Cahiers du français contemporain, 3, 77-90 (1996)

4. D. Caillat, Le discours rapporté direct dans les conversations orales: un système de balisage multimodal. In C. Desoutter, C. Mellet (éds.) Le discours rapporté et ses marques, 63-80 (Peter Lang, Bern, 2013)

5. H. Sacks, E. A. Schegloff, G. Jefferson, A simplest systematics for the organization of turn-taking for conversation. Language, 50, 4, 696-737 (1974)

6. V. Traverso, L'analyse des conversations. (Nathan, Paris, 1999)

7. C. Petitjean, S. Pekarek Doehler, Développemets actuels en analyse conversationnelle et recherches sur les interactions en français. Revue française de linguistique appliquée, 22, 2, 5-14 (2017)

8. L. Mondada, Nouveaux défis pour l'analyse conversationnelle : l'oganisation située et systématique de l'interaction sociale. Langage et Société, 160-161, 2, 181-197 (2017)

9. L. Mondada, Transcription in linguistics. In L. Litosseliti (éd.) Research Methods in linguistics : Second Edition, 85-114 (Bloomsbury, London, 2018)

10. L. Rosier, Le discours rapporté: Histoire, théories, pratiques. (Duculot, ParisBruxelles, 1999)

11. C. Goodwin, Participation, Stance and Affect in the Organization of Activities. Discourse and Society, 18, 1 (2007) 
12. K. Stec, M. Huiskes, G. Redeker, Multimodal analysis of quotation in oral narratives. Open Linguistics, 1, 1 (2015)

13. L. Mondada, S. Keel, Participation et asymétries dans l'interaction institutionnelle. (L'Harmattan, Paris, 2017)

14. M. Grosjean, Les relèves orales entre équipes infirmières à l'hôpital. Des structurations discursives aux pratiques cognitives et sociales. In M. Grossen, B. Py (éds.) Pratiques sociales et médiations symboliques, 137-52 (Peter Lang, Bern, 1997)

15. M. Grosjean, From multi-participant talk to genuine polylogue: shift-change briefing sessions at the hospital. Journal of Pragmatics, 36, 1, 25-52 (2004)

16. M. Grosjean, M. Lacoste, Communication et intelligence collective. (PUF, Paris, 1999)

17. I. Colón de Carvajal, L. Maritaud, B. Chalancon, De la transmission d'informations cliniques au partage de savoir lors de relèves infirmières. TIPA, 36 (accepté)

18. A. Kendon, Some functions of gaze-direction in social interaction. Acta Psychologica, 26, 22-63 (1967)

19. A. Kendon, M. Cook, The consistecy of gaze patterns in social interaction. British Journal of Psychology, 60, 4, 481-494 (1969)

20. L. Mondada, La constitution de l'origo déictique comme travail interactionnel des participants : une approche praxéologique de la spatialité. Intellectica, 41-42, 75-100 (2005)

21. L. Mondada, Multimodal resources for turn-taking: Pointing and the emergence of possible next speakers. Discourse Studies, 9 , 2 , 195-226 (2007)

22. A. Mazur-Palandre, I. Colón de Carvajal, Multimodalité du langage dans les interactions et l'acquisition. (UGA éditions, renoble, 2020)

23. G. Jefferson, List-construction as a task and a resource. In G. Psathas (éd.) Interactional Competence, 63-92 (University Press of America, Washington, DC, 1990)

\section{Annexes}

\section{Conventions de transcription}

$\begin{array}{ll}\text { [ ] } & \text { Chevauchement de paroles } \\ \text { / I } & \text { Intonations montantes ou descendantes } \\ \text { : : : } & \text { Allongement d'un son ou d'une syllabe } \\ \text { p'tit } & \text { Elision } \\ \text { trouv- } & \text { Troncation d'un mot } \\ \text { (inaud.) } & \text { Partie incompréhensible } \\ \text { ( ) } & \text { Transcription incertaine } \\ \text { (.) } & \text { Micro-pause } \\ \text { \& } & \text { Tour s'étend sur plusieurs lignes } \\ \text { ( } & \text { Murmuré }\end{array}$

Plus de détails : http://icar.univ-

lyon2.fr/projets/corinte/documents/2013_Conv_ICOR_250313.pdf

La transcription de la parole est en police noire

La transcription multimodale est en police grise

Les conventions de transcription de la multimodalité sont reprises de Mondada (2018) :

Les descriptions multimodales sont temporellement alignées aux tours de parole à l'aide de symbole. 
@ ou $£$ ou $\$$ Un symbole est associé à chaque locuteur pour décrire les regards et les gestes réalisés lorsqu'ils sont pertinents pour l'analyse.

@regarde GIL@ On note le début et la fin du phénomène multimodal par le même symbole.

$-->$ Si le phénomène multimodal se prolonge sur plusieurs tours. On indique parfois le numéro de la ligne correspondante à la fin du

\# phénomène (ex : - ->45).

Les images illustrant un phénomène multimodal sont notées par ce symbole qui est

temporellement aligné au verbal. 